\title{
Eccentricity of Selected Eclipsing Systems
}

\author{
W. Ogłoza ${ }^{1}$, J. M. Kreiner ${ }^{1}$, G. Stachowski ${ }^{1}$, B. Zakrzewski ${ }^{1}$, \\ Z. Mikulášek ${ }^{2}$, and M. Zejda ${ }^{2}$ \\ ${ }^{1}$ Mt. Suhora Observatory, Cracow Pedagogical University, \\ 30-084 Cracow, ul Podchorążych 2, Poland \\ email: ogloza@up.krakow.pl \\ ${ }^{2}$ Masaryk University, Brno, Czech Republic
}

\begin{abstract}
This paper presents the results of verification of known stars showing evidence of orbital eccentricity and apsidal motion.
\end{abstract}

Keywords. stars: binaries: eclipsing, ephemerides

\section{Introduction}

The study of orbital eccentricity and apsidal motion are very important for verification of theories of evolution of binary systems and of the internal structure of stars. One of the methods used to study long time effects is (O-C) analysis of times of minima.

This paper presents the preliminary results of the selection of GCVS eclipsing systems with phase-shifted secondary minima. At present, the Cracow database contains 194993 individual minima of 5931 stars. Examination of the (O-C) diagrams shows about 256 stars with a significant phase shift of the secondary minimum, interpreted as the effect of an eccentric orbit. The poster shows 98 selected systems (with 5800 times of minima) for which the primary and secondary minima were observed at least 4 times over well separated epochs (e.g. the diagram for V799 Cas). About $56 \%$ of those systems show the effect of rotation of the line of apsides (see the diagram for the well known system Y Cyg).

\section{Results}

The orbital period of the eccentric orbit binaries is in the range of $\sim 1.2$ to $\sim 20$ days (except OW Gem $P \approx 1200 \mathrm{~d}$ V883 Mon $P \approx 50 \mathrm{~d}$, VW Peg $P \approx 21 \mathrm{~d}$, and LV Her $P \approx 18 \mathrm{~d}$ ), but for long-period systems the number of minima is small.

There are a few short-period variables which show an apparent phase shift of the secondary minimum (LV Vir $P=0.4094$, TY Men $P=0.4617$, V407 Peg $P=0.6369$, LR Car $P=0.8529$, GI Boo $P=1.0335$, DU Boo $P=1.0559)$, however this could be the effect of an asymmetrically-shaped minimum.

For several systems (V947 Cyg, RW Lac, RU Mon, $\zeta$ Phe, V1094 Tau, AO Vel, DR $\mathrm{Vul}$ ) the effect of an eccentric orbit on the (O-C) diagram is present together with other phenomena, such as constant period change or the influence of a third body.

\section{Database}

The full list of eccentric systems, pictures of O-C diagrams and up-to-date linear elements for primary and secondary minima will be continuously published at:

www.as.up.krakow.pl/ephem 


\section{Reference}

Kreiner, J. M. 2004, AcA, 50, 247 